\title{
In vitro Evaluation of 2-/3-Alkoxyphenylcarbamic Acid Esters Containing N-Arylpiperazine Moiety Against Some Nontuberculous Mycobacteria
}

\author{
Ivan Malik ${ }^{1}$, Iveta Zadrazilova² and J osef J ampilek ${ }^{2}$ \\ ${ }^{1}$ Department of Pharmaceutical Chemistry, Faculty of Pharmacy, Comenius University, Odbojárov 10, 83232 \\ Bratislava, Slovak Republic \\ ${ }^{2}$ Department of Chemical Drugs, Faculty of Pharmacy, University of Veterinary and Pharmaceutical Sciences \\ Palackého 1/3, 61242 Brno, Czech Republic
}

Received: August 08, 2015; Accepted: October 19, 2015; Published (web): January 31, 2016

\begin{abstract}
Nontuberculous mycobacteria (NTM) are a diverse group of ubiquitous environmental organisms and include more than 125 identified species broadly classifiable into "slow" and"rapid" growers. NTM are present in the environment, mainly in water, and are occasionally responsible for opportunistic infections in humans. Despite the fact that NTM are characterized by a moderate pathogenicity, the diseases caused by NTM at various body sites are increasing worldwide. ${ }^{1,2}$ Pulmonary involvement is the most common disease manifestation of NTM. ${ }^{3,4}$ Furthermore, patients with cystic fibrosis, the most frequent lethal genetic disorder of White persons, face numerous infectious pathogens over the course of their lifespan, increasing attention has recently been paid to NTM. ${ }^{5,6}$

Treatment of infections caused by NTM is either by drug therapy, surgery, or both. Drug therapy of NTM diseases is long, costly, and often associated with drug-related toxicities. The treatment regimens differ by species with the most important distinction being that between slow and rapid growing NTM. ${ }^{7}$
\end{abstract}

Correspondence to: Ivan Malik

Tel.: +421-2-50 117 226; Fax: +421-2-50 117 100;

E-mail: malikivan001@gmail.com

Dhaka Univ. J. Pharm. Sci. 14(20): 231-234, 2015 (December)
Searching for novel structures that NTM have very rarely been presented with before, the aim of current research is to in vitro investigate the efficiency of 2-/3-alkoxyphenylcarbamic acid esters containing 4-(pyrimidin-2-yl) piperazin-1-yl fragment within their structure (Table 1) against some members of NTM strains, namely Mycobacterium marinum CAMP 5644, M. kansasii DSM 44162 and M. smegmatis ATCC 700084 and try to identify some structural features which could be beneficial in terms of their potency. The molecules 1-4 have been previously investigated against Grampositive bacteria Staphylococcus aureus ATCC 6538 (Micrococcaceae), Gram-negative bacteria $\begin{array}{llll}\text { Escherichia coli } & \text { CNCTC } & 377 / 79\end{array}$ (Enterobacteriaceae) and yeast Candida albicans CCM 8186 as well. ${ }^{8}$

The derivatives under the study 1-4 (Table 1), chemically 1-[3-(2-/3-alkoxyphenylcarbamoyloxy)-2hydroxypropyl]-4-(pyrimidin-2-yl)piperazinium chlorides (where alkoxy=methoxy or ethoxy), were obtained from Department of Pharmaceutical Chemistry, Faculty of Pharmacy, Comenius University in Bratislava, Slovak Republic.

The evaluation of in vitro antimycobacterial activity of the compounds was performed against $M$. marinum CAMP 5644, M. kansasii DSM 44162 and M. smegmatis ATCC 700084. The broth dilution 
micromethod in Middlebrook 7H9 medium (Difco, USA) supplemented with BD BBL Middlebrook ADC Enrichment (Becton, USA) was used to determine the minimum inhibitory concentration (MIC) as previously described. ${ }^{9}$ The compounds were dissolved in dimethyl sulfoxide (DMSO, Sigma-Aldrich, Germany), and the final concentration of DMSO did not exceed $2.5 \%$ of the total solution composition. The final concentrations of the evaluated compounds ranging from 256 to $0.125 \mu \mathrm{g} / \mathrm{mL}$ were obtained by two-fold serial dilution of the stock solution in microtiter plate with sterile medium. Isoniazid (INH; Sigma-Aldrich, Germany) and rifampicin (RIF; Sigma-Aldrich, Germany) were used as the standards as they are clinically used antimycobacterial drugs. Bacterial inocula were prepared by transferring colonies from culture to sterile water. The cell density was adjusted to $0.5 \mathrm{McF}$ arland units using a densitometer (Densi-
La-Meter, LIAP, Latvia). The final inoculum was made by $1: 1000$ dilution of the suspension with sterile water. Drug-free controls, sterility controls and controls consisted of medium and DMSO alone were included. The determination of results was performed visually after 3 days of static incubation in the darkness at $37^{\circ} \mathrm{C}$ in an aerobic atmosphere for $M$. smegmatis, after 7 days of static incubation in the darkness at $37^{\circ} \mathrm{C}$ in an aerobic atmosphere for $M$. kansasii and after 21 days of static incubation in the darkness at $28{ }^{\circ} \mathrm{C}$ in an aerobic atmosphere for $M$. marinum. ${ }^{10}$

The MICs were defined as the lowest concentration of the compound at which no visible bacterial growth was observed. The MIC readout is routinely and widely used in bacterial assays and is a standard detection limit according to the Clinical and Laboratory Standards Institute (CLSI, www.clsi.org). The results are summarized in table 1 .

Table 1. Activity of the in vitro investigated compounds 1-4 against some nontuberculous mycobacterial strains expressed by $M I C$ values $(\mu \mathrm{mol} / \mathrm{l})$.

\begin{tabular}{ccccc}
\hline & & \\
\hline \\
\hline
\end{tabular}

To be the best of authors' knowledge, current paper presents the first report dealing with the activity of 2-/3-alkoxyphenylcarbamic acid esters containing 2-hydroxypropane-1,3-diyl connecting chain and $N$-arylpiperazine group against $M$. marinum CAMP 5644, M. kansasii DSM 44162 and M. smegmatis ATCC 700084 as well.
Following chemical structure of currently in vitro investigated compounds 1-4 (Table 1), their potency against given NTM might be influenced, among others, by: (i) 2-/3-alkoxy side chain isomerism, (ii) their lipohydrophilic profile, (iii) nature of salt forming moiety. Possible impacts of 
aforementioned factors will be discussed in the parts below.

As current results clearly indicated (Table 1), positional isomerism of alkoxy group did not affect the potency of the derivatives under the study against all the inspected mycobacteria. In terms of 2-alkoxy substituted derivatives, proximity of attached 2alkoxy chain to carbamate bond led to the twist of the phenyl ring plain towards carbamate group. Described process resulted in the planarity violation of such molecule(s) to provide subsequent conjugation of $\pi$-bonds of aromatic ring over $\mathrm{NH}$ fragment up to carbonyl group. The outcome of this conjugation was different electronic density (charge) at carbonyl moiety which could be one of possible binding sites to reactive membrane locations. In the series of 3-alkoxy substituted compounds, given secondary steric effect did not manifest. Current results confirmed that described notable intramolecular interactions did not influenced the activity. Following given preliminary data, it was not clear if such lipophilic moiety (alkoxyphenyl group) could be regarded as essential part of pharmacophore. Taking into account effect of the positional isomerism, further experimental determinations will be carried out for more comprehensive conclusions.

All the investigated derivatives were considered more efficient against M. marinum than INH. Furthermore, elongation of attached alkoxy side chain in both series of 2- and 3-substituted molecules, i.e. the increase in lipophilicity, led to more promising results for M. kansasii strain only. On the other hand, both compounds $\mathbf{2}$ and $\mathbf{4}$ have shown higher MIC outputs than used standards INH and RIF not only for M. kansasii but also for M. smegmatis. The standard RIF were regarded as the most active compound of entire tested set against all in vitro inspected NTM (Table).

In general, it seemed that lipophilicity could be important factor affecting the potency of alkoxyphenylcarbamic acid esters containing 2hydroxypropane-1,3-diyl and $N$-arylpiperazine moiety not only against given Mycobacterium but also against previously in vitro tested $M$. tuberculosis $\mathrm{H}_{37} \mathrm{R}_{\mathrm{v}}$ and C. albicans. ${ }^{11,12}$

Furthermore, it could be suggested that the differences in assumed electronic and hydrophobic interactions with effector sites of tested NTM involved by various substituents attached to piperazin-1,4-diyl would be decisive for the activity of thus alkoxyphenylcarbamic acid-based substances. Following conclusions from previous research, the presence of pyrimidin-2-yl in the chemical structure of $\mathrm{N}$-arylpiperazine compounds would be favorable in terms of their toxicological profile due to formation of 1-(pyrimidin-2-yl) piperazine, an active metabolite, which is converted to 5-hydroxy-1(pyrimidin-2-yl) piperazine in human liver microsomes. ${ }^{13,14}$ On the other hand, both atoms of nitrogen have shown the ability to polarize the $\pi$ electron system of pyrimidin-2-yl, resulting in slightly decreased electron density on the ring carbons. It would be probable that incorporation of (suitably substituted) phenyl instead of heterocyclic pyrimidin-2-yl would lead to more promising results.

\section{ACKNOWLEDGEMENTS}

The authors are very grateful to Slovak Grant Agency for Science for supporting by the VEGA Grant Project No. 1/0029/16 and to Internal Grant Agency of University of Veterinary and Pharmaceutical Sciences in Brno for supporting by the Grant Project No. 304/2015/FaF. The authors thank anonymous reviewers for their valuable comments and helpful revision suggestions.

\section{REFERENCES}

1. Tortoli, E. 2014. Microbiological features and clinical relevance of new species of the genus Mycobacterium. Clin. Microbiol. Rev. 27, 727-752.

2. Griffith, D.E., Aksamit, T., Brown-Elliot B.A., Catanzaro, A., Daley, Ch., Gordin, F., Holland, S.M., Horsburgh, R., Huitt, G., Iademarco, M.F., Iseman, M., Olivier, K., Ruoss, S., von Reyn, C.F., Wallace Jr., R.J. and Winthrop, K. 2007. An official ATS/IDSA statement: diagnosis, treatment, and prevention of nontuberculous mycobacterial diseases. Am. J. Respir. Crit. Care Med. 175, 367-416. 
3. Hoefsloot, W., van Ingen, J., Andrejak, C., Ängeby, K., Bauriaud, R., Bemer, P., Beylis, N., Boeree, M.J., Cacho, J., Chihota, V., Chimara, E., Churchyard, G., Cias, R., Daza, R., Daley, Ch.L., Dekhuijzen, P.N.R., Domingo, D., Drobniewski, F., Esteban, J., Fauville-Dufaux, M., Folkvardsen, D.B., Gibbons, N., Gómez-Mampaso, E., Gonzalez, R., Hoffmann, H., Hsueh, P.-R., Indra, A., Jagielski, T., Jamieson, F., Jankovic, M., Jong, E., Keane, J., Koh, W.-J., Lange, B., Leao, S., Macedo, R., Mannsåker, T., Marras, T.K., Maugein, J., Milburn, H.J., Mlinkó, T., Morcillo, N., Morimoto, K., Papaventsis, D., Palenque, E., Paez-Pena, M., Piersimoni, C., Polanová, M., Rastogi, N., Richter, E., Ruiz-Serrano, M.J. Silva, A., da Silva, M.P., Simsek, H., van Soolingen, D., Szabó, N., Thomson, R., Tórtola Fernandez, T., Tortoli, E., Totten, S.E., Tyrrell, G., Vasankari, T., Villar, M., Walkiewicz, R., Winthrop, K.L. and Wagner, D., for the Nontuberculous Mycobacteria Network European Trials Group (NTM-NET). 2013. The geographic diversity of nontuberculous mycobacteria isolated from pulmonary samples. Eur. Respir. J. 42, 1604-1613.

4. Ebrahimi, G., Farshidpour, M., Allen, M.B., Mirsaeidi, M. and Falkinham, J.O. 2015. Nontuberculous mycobacterial disease in North America. Int. J. Mycobacteriol. 4, 154-155.

5. Qvist, T., Gilljam, M., Jönsson, B., Taylor-Robinson, D., Jensen-Fangel, S., Wang, M., Svahn, A., Kötz, K., Hansson, L., Hollsing, A., Hansen, Ch.R., Finstad, P.L., Pressler, T., Høiby, N. and Katzenstein, T.L. 2015. Epidemiology of nontuberculous mycobacteria among patients with cystic fibrosis in Scandinavia. J. Cyst. Fibros. 14, 46-52.

6. Seddon, P., Fidler, K., Raman, S., Wyatt, H., Ruiz, G., Elston, C., Perrin, F., Gyi, K., Bilton, D., Drobniewski, F. and Newport, M. 2013. Prevalence of nontuberculous mycobacteria in cystic fibrosis clinics, United Kingdom, 2009. Emerg. Infect. Dis. 19, 1128-1130.

7. van Ingen, J., Boeree, M.J., van Soolingen, D. and Mouton, J.W. 2012. Resistance mechanisms and drug susceptibility testing of nontuberculous mycobacteria. Drug Resist. Update 15, 149-161
8. Malik, I., Sedlarova, E., Bukovsky, M., Csollei, J., Stanzel, L. and Sichrovska, L. 2014. In vitro antimicrobial evaluation of ortho-/meta-alkoxyphenylcarbamic acid esters containing 4-(pyrimidin-2-yl)piperazin-1-yl moiety. Dhaka Univ. J. Pharm. Sci. 13, 215-219.

9. Schwalbe, R., Steele-Moore, L. and Goodwin, A.C. 2007. Antimicrobial Susceptibility Testing Protocols. CRC Press, Boca Raton, p. 432.

10. Gonec, T., Kos, J., Zadrazilova I., Pesko, M., Keltosova, S., Tengler, J., Bobal, P., Kollar, P., Cizek, A., Kralova, K. and Jampilek, J. 2013. Antimycobacterial and herbicidal activity of ring-substituted 1-hydroxynaphthalene-2-carboxanilides. Bioorg. Med. Chem. 21, 6531-6541.

11. Waisser, K., Doležal, R., Čižmárik, J., Malík, I. and Kaustová, J. 2007. The potential antituberculotics of the series of 2-hydroxy-3-(4-phenylpiperazin-1-yl)propylphenylcarbamates. Folia Pharm. Univ. Carol. 35-36, 45-48.

12. Malík, I., Bukovský, M., Andriamainty, F. and Gališinová, J. 2012. Antimicrobial activity of metaalkoxyphenylcarbamates containing substituted $\mathrm{N}$ phenylpiperazine fragment. Braz. J. Microbiol. 43, 959-965.

13. Kalgutkar, A.S., Vaz, A.D.N., Lame, M.E., Henne, K.R., Soglia, J., Zhao, S.X., Abramov, Y.A., Lombardo, F., Collin, C., Hendsch, Z.S. and Hop, C.E.C.A. 2005. Bioactivation of the nontricyclic antidepressant nefazodone to a reactive quinone-imine species in human liver microsomes and recombinant cytochrome P450 3A4. Drug Metab. Dispos. 33, 243-253.

14. Raghavan, N., Zhang, D., Zhu, M., Zeng, J. and Christopher, L. 2005. CYP2D6 catalyzes 5-hydroxylation of 1-(2pyrimidinyl)-piperazine, an active metabolite of several psychoactive drugs, in human liver microsomes. Drug Metab. Dispos. 33, 203-208. 